\title{
From Virtuality to Reality Social Media Facilitates the Interaction Between Global Microsurgeons
}

\author{
Jennifer An-Jou Lin, MD'; Usama Farghaly Omar, ${ }^{1} D^{2}$; \\ Musa Mateev, MD, PhD³; Tommy Nai-Jen Chang, $\mathrm{MD}^{1 *}$
}

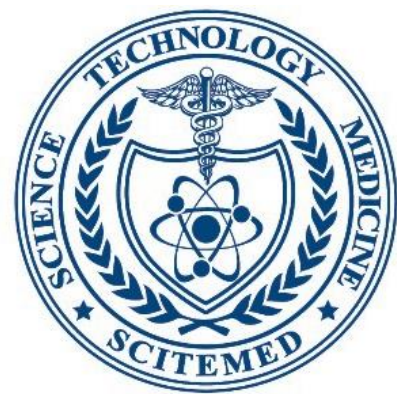

1Division of Reconstructive Microsurgery, Department of Plastic and Reconstructive Surgery, Chang Gung Memorial Hospital, Linkou Medical Center and Chang Gung Medical College and Chang Gung University, Taoyuan, Taiwan

2Department of Orthopaedic Surgery, Al-Hekma Specialized Hospital, Assiut, Egypt

${ }^{3}$ Department of Plastic and Reconstructive Surgery, Kyrgyz-Slavian International University, Bishkek, Kyrgyz Republic

W ith the widespread use of the internet, social media nowadays plays a significant role in our lives, from daily activities to educational access and work fields. It has revolutionized not only the personal communication but also the interaction among professionals $[1,2]$. In the medical field, social media such as Facebook, Twitter, Blogs, Line, and WeChat provide abundant usages for communication among staff, meetings, medical education and conferences [3]. International Microsurgery Club (IMC) [4], an invited-only group based on Facebook, has shown to us a successful example in the fields of microsurgery. It was established in May 2016, aiming to provide a convenient forum to discuss and share about challenging cases and resources for global mi-

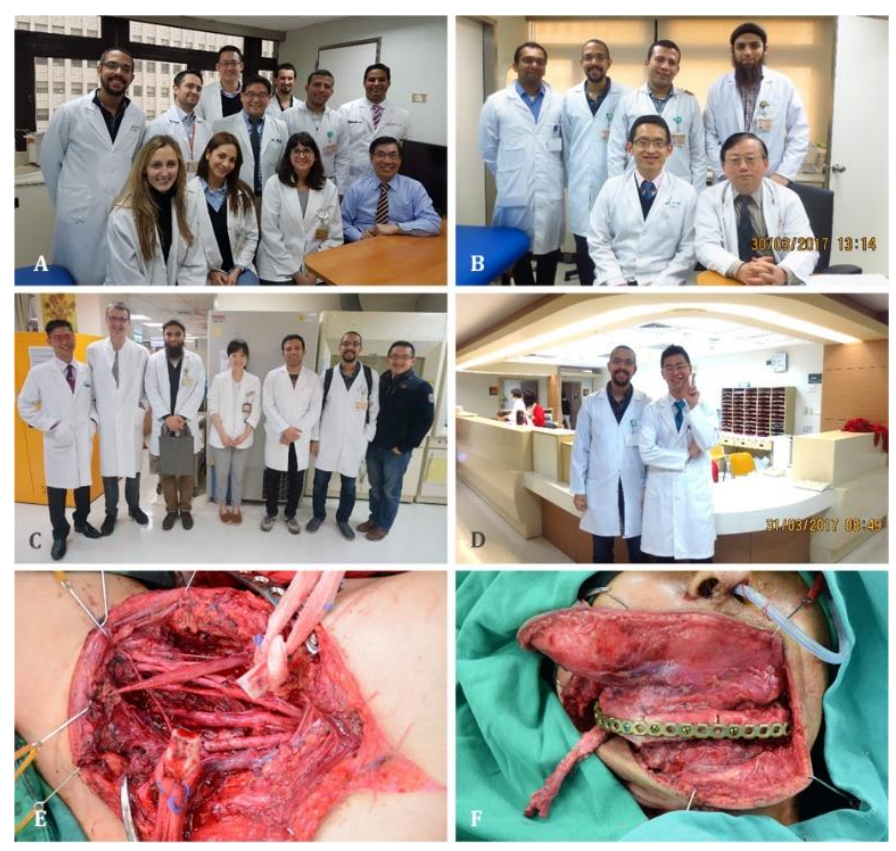

Figure 1. Dr. Usama Farghaly Omar (Egypt) visited Chang Gung Memorial Hospital, Taiwan. (A) Joining Prof. Fu-Chan Wei's outpatient department. (B) Joining Prof. David Chwei-Chin Chuang and Dr. Johnny Chuieng-Yi Lu's outpatient department. (C) Visiting the common laboratory of the Division of Reconstructive Microsurgery, Department of Plastic and Reconstructive Surgery. (D) Meeting with Dr. Tommy Nai-Jen Chang, the funder and the primary investigator of International Microsurgery Club (IMC). (E) Brachial plexus exploration in Chang Gung Memorial Hospital. (F) Mandible reconstruction via a fibula osteoseptocutaneous flap after the oncologic resection in Chang Gung Memorial Hospital. crosurgeons [5]. Until October 2017, the memberships have grown to more than 6400 members and it has become one of the largest and the most active online microsurgery platforms in the world.

Since the emergence of IMC, the interaction and communication among international microsurgeons have become more convenient and diverse. Firstly, the learning switches from one direction (from paper, textbook) to two-way interaction, or even multiway interaction. When a case or a question is released on the page, the members can either discuss through the comments below, or contact via messages. Secondly, it makes the discussion between one another more straightforward. Members are able to connect with other surgeons directly by sending messages or posting on the IMC page. The third characteristic is the instantaneity. Through the Internet, microsurgeons from different time zones are able to set a discussion without any time constraint. Besides, all the posts can be followed up and reviewed later. Last but not least, there are some microsurgeons who haven't published a lot, but have a bunch of clinical experiences; therefore, they can post their masterpieces on IMC with the easy form; and so many "no-name heroes" have been identified, who were not familiar to the global microsurgeons before [5].

The impact wasn't limited to the cyber world. IMC was just the catalytic agent since the members still wanted to know others face-to-face eventually. After knowing from the online platform, some of them proposed for opportunities such as visiting scholarship, fellowship, or meet

$n=63$

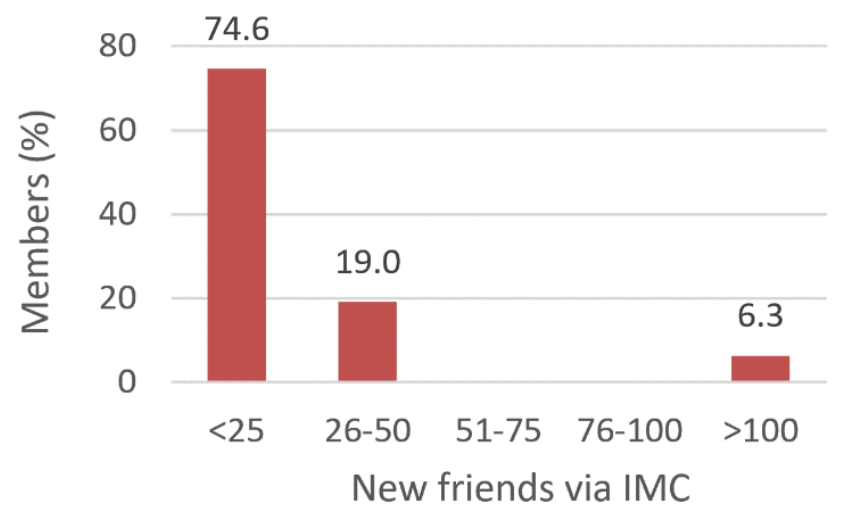

Figure 2. Among 63 respondents, $74.6 \%$ had made less than 25 new friends on Facebook through IMC; $19.0 \%$ got more than 25 friends; and $6.3 \%$ had more than 100 friends. 
in the conferences. There were two previous surveys we obtained in the IMC group related to the interaction among the members. We queried that how many Facebook friends one had made through IMC since it was established (May 2016) and whether one had ever met the members of IMC in person (either visiting someone or visited by someone) through the connection of IMC. Among 63 respondents, 19.0\% got more than 25 friends on Facebook and 6.3\% had more than 100 friends (Figure 2). In the second survey, $30.4 \%$ had met the other IMC members in real life already and $39.1 \%$ members hoped to meet soon in the future (Figure 3 ).

\section{$n=69$}

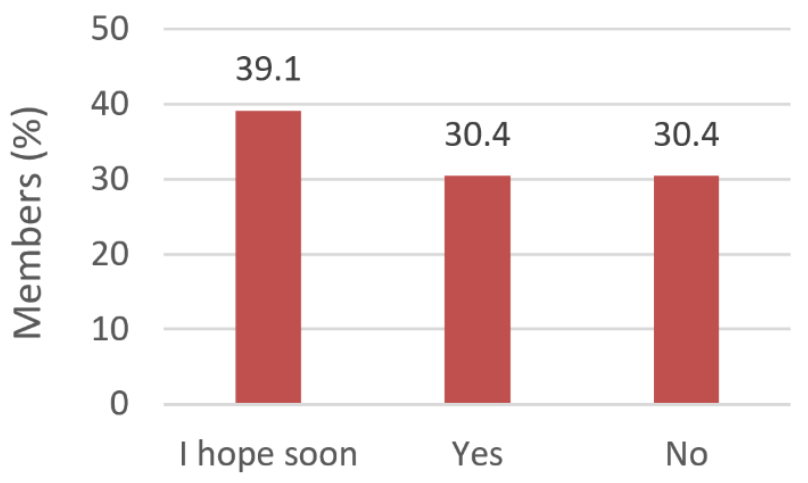

Figure 3. Among 69 respondents, $30.4 \%$ had met other IMC members and $39.1 \%$ members hoped they would meet soon in the future.

\section{International Scholars in Chang-Gung}

Since the end of 2016 until now (October 2017), there have been around ten microsurgeons visiting Chang Gung Memorial Hospital, Linkou Medical Center, Taiwan by the introduction of the IMC. Dr. Usama Farghaly Omar from Egypt visited us between January and March 2017 and shared his precious experience with us (Figure 1).

"Being an orthopedic surgeon interested in microsurgery, I was looking forward to deepening my experience by visiting one of the international centers for microsurgery. I got the chance to visit Chang Gung Memorial Hospital in Taiwan, one of the leading and most innovative microsurgery centers in the world. During the period from January 2017 to March 2017, I was exposed to different subspecialties of reconstructive microsurgery as head and neck reconstruction, peripheral nerve surgery, upper and lower extremity reconstruction. I mainly followed Prof. David Chwei-Chin Chuang and his team, as I was eager to more knowledge about brachial plexus and peripheral nerve surgery. I've been able to observe different techniques of management of different case scenarios, besides their diagnosis and follow up in clinic. I've been allowed to share in some research projects. I really enjoyed this visitorship and would strongly recommend it for all surgeons who were interested in microsurgery. This opportunity wouldn't be with that ease, unless the barriers had been broken with social media that made communication and getting in touch with world experts so easy. The great idea made by Dr. Tommy Nai-Jen Chang, International Microsurgery Club, introduced us to a new era of gaining universal experience from nearly all centers of the world."

\section{Chang-Gung International Scholars}

As one of the members of IMC from Taiwan and a resident having great fond of microsurgery, I had an extraordinary experience to visit Professor Musa Mateev in Kyrgyzstan by the bridge of IMC in the summer of 2017. During this visit, Prof. Mateev introduced his clinic to me in person, from the operative rooms to the wards and the clinic room. Besides, he gave me a comprehensive lecture about the perforator flaps and aesthetic cases he had operated (Figures 4-6). He shared with me his 30-more-yearexperience as a plastic surgeon and his values of life as a mentor. Since I was just a first-year-resident, he gave me many constructive advices about my future. I learned from him the ways to successfully manage a clinic. Also, this was the first time I was taught thoroughly about the perforator flaps and how widely it was used in his hospital compared to our

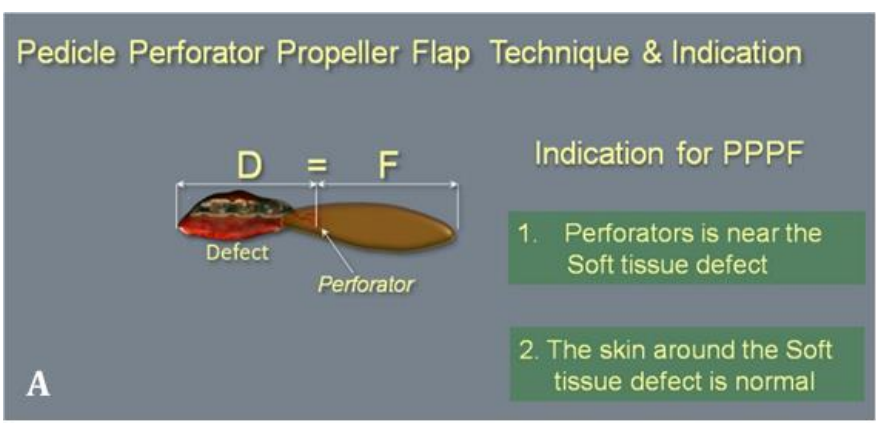

Pedicle Perforator Propeller Flap Technique \& Indication

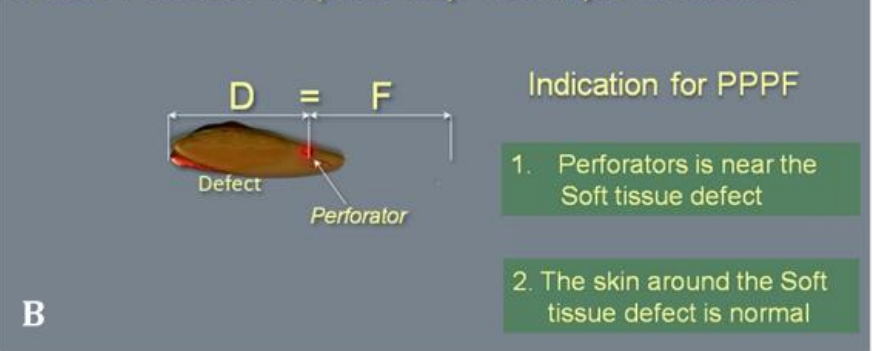

Figure 4. Illustration for the technique and the indication of perforator propeller flap (PPF) by Dr. Musa Mateev. If the perforator is near the soft tissue defect, or the skin around the soft tissue defect is normal, a perforator propeller flap is suitable for reconstruction. There are several advantages of PPF. First, the color and the texture of the flap is same as the soft tissue around the recipient site. Second, the flap is thin. Third, the operative time is short and only one team of surgeons is needed (neither microanastomosis nor distant flap).

region. Importantly, through this unique experience, I gained numerous fresh perspectives due to the different culture, medical policy, and the local conditions and discovered the variety and innovations in microsurgery through countries to countries. Were it not for the connection made by Dr. Tommy Nai-Jen Chang, the founder of IMC, I could not have this meaningful experience adding to my trip (Figure 7).

\section{The IMC Members' Reunion in the International Meetings}

Through the Internet, knowledge can spread beyond the boundaries and distance. Linkages have been built up on the online social media. Nowadays, more and more microsurgery meetings start to set up the social media section to discuss the details and try to push the effect to the limit. The senior author (Dr. Tommy Nai-Jen Chang) and a part of his group members (Dr. Soo-Ha Kwon, Dr. Angela Ting-Wei Hsu, Dr. Evelyn Ting-

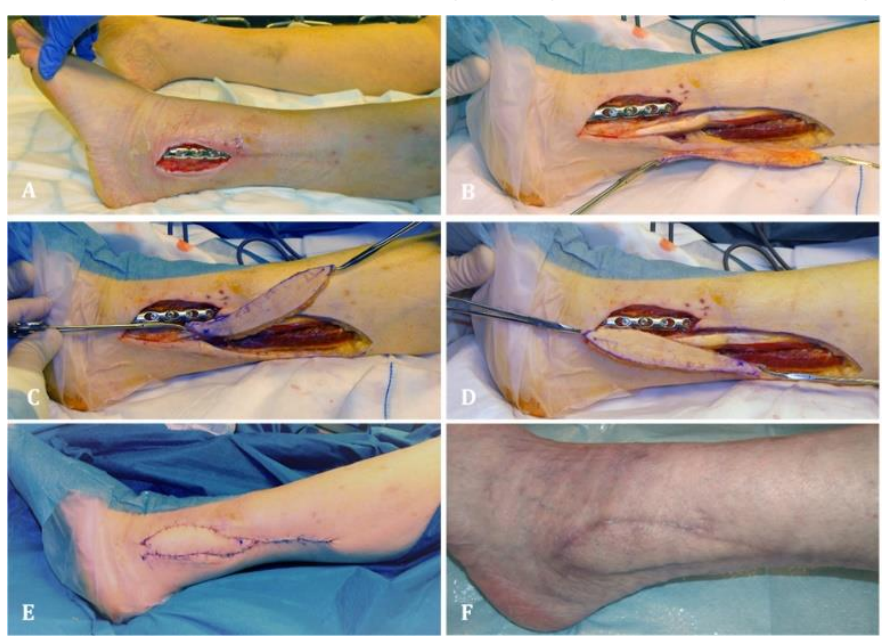

Figure 5. A case of fibula artery perforator propeller flap (FAPPF) (Demonstrated by Dr. Musa Mateev). (A) This 47-year-old female patient with the history of traumatic open fracture due to traffic accident three months ago presented infected soft tissue defect (size $7 \times 3 \mathrm{~cm}$ ) on the lateral left ankle. (B) The FAPPF was harvested. (C and D) FAPPF was rotated 180 degrees. (E) Donor site was closed primarily. (F) Post-operative pictures of recipient and donor sites. 


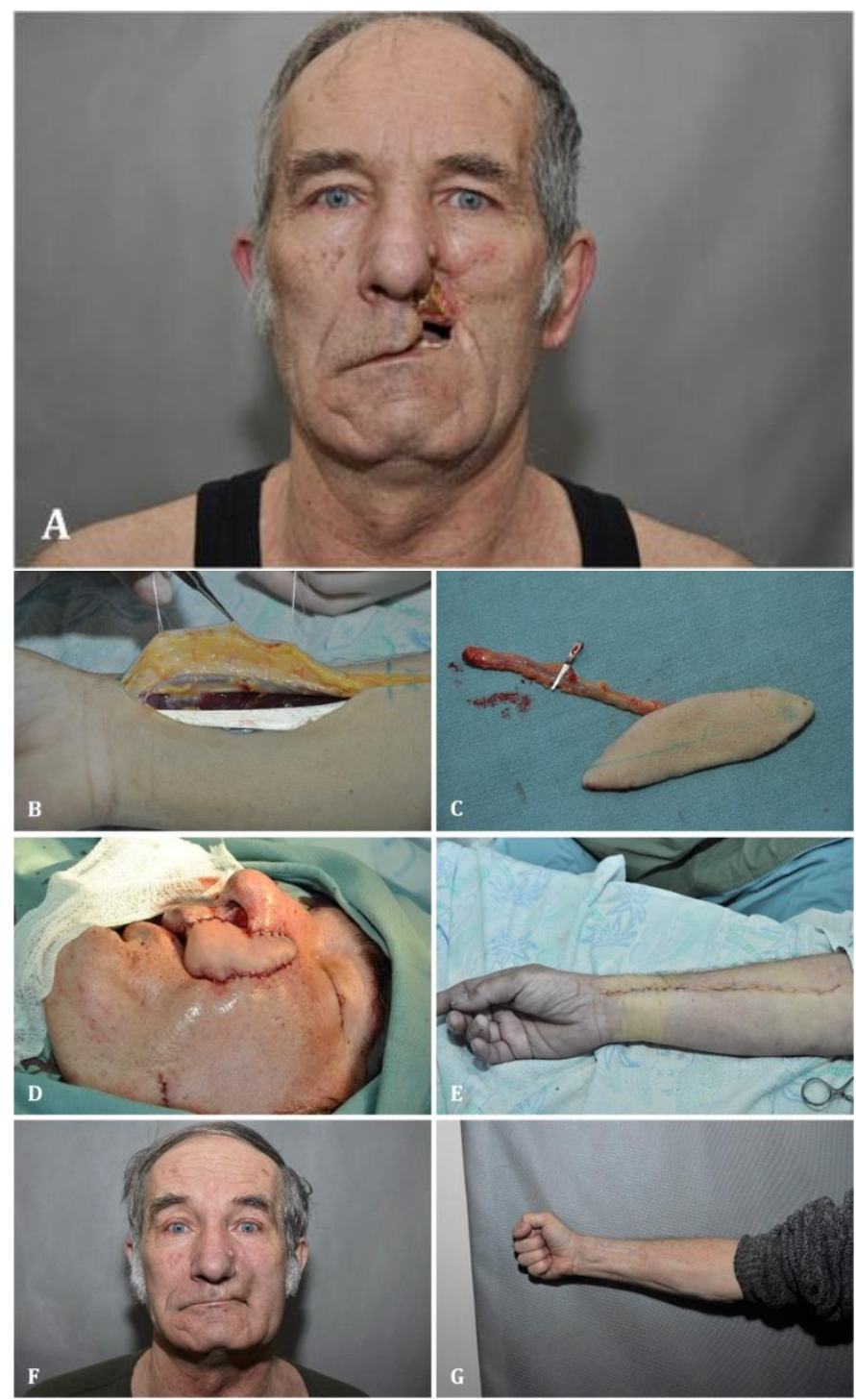

Figure 6. A case of radial artery perforator flap (Demonstrated by Dr. Musa Mateev). (A) This 62-year-old male patient with the history of post operation with wide tumor resection 6 months ago had suffered from skin infection and necrosis postoperatively. As a result, he presented a soft tissue defect of size $5 \times 3 \mathrm{~cm}$. (B and C) The radial artery perforator flap was harvested. (D) Microvascular anastomosis was performed with the facial artery and vein as recipient artery and vein, respectively. $(E)$ Donor site was closed primarily. ( $F$ and $G$ ) Post-operative pictures of recipient and donor sites.

Hsuan Tang) have been invited to the World Society Reconstructive Microsurgery (WSRM) meeting, International Microsurgical Simulation Society (IMSS, June 2017 in Seoul, Korea) and International Course on Super Microsurgery meeting (ICSM, October 2017 in Jinan, China) for the penal talk. We really appreciate the opportunity provided by Dr. Joon-Pio Hong, Dr. Daniel Liu, Dr. Ali Ghanem, and Dr. Zengtao Wang (Figure 8). We believe that the online platform will play an immensely important role in the future and the global microsurgeons will benefit from it. The impact is not only on the Internet but also in the real world - from virtuality to reality.

\section{Article Information}

*Correspondence: Tommy Nai-Jen Chang, MD

Division of Reconstructive Microsurgery, Department of Plastic and Reconstructive Surgery, Chang Gung Memorial Hospital, No. 5, Fu-Hsing St. Kwei-Shan, Taoyuan, Taiwan. E-mail: tommynjchang@yahoo.com.tw

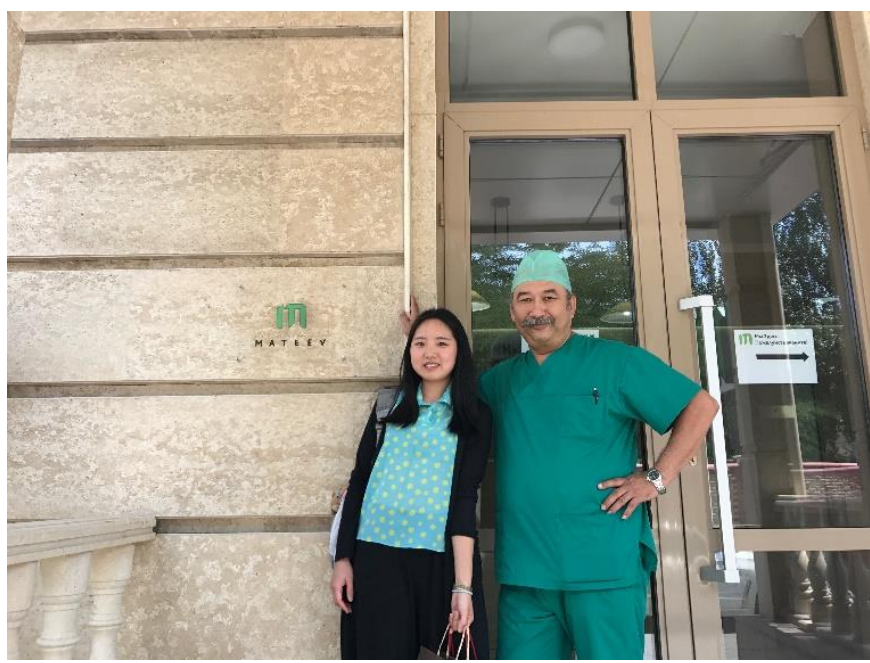

Figure 7. The junior author Jennifer An-Jou Lin (Taiwan) visited Prof. Musa Mateev in Kyrgyzstan by the bridge of IMC in the summer of 2017.

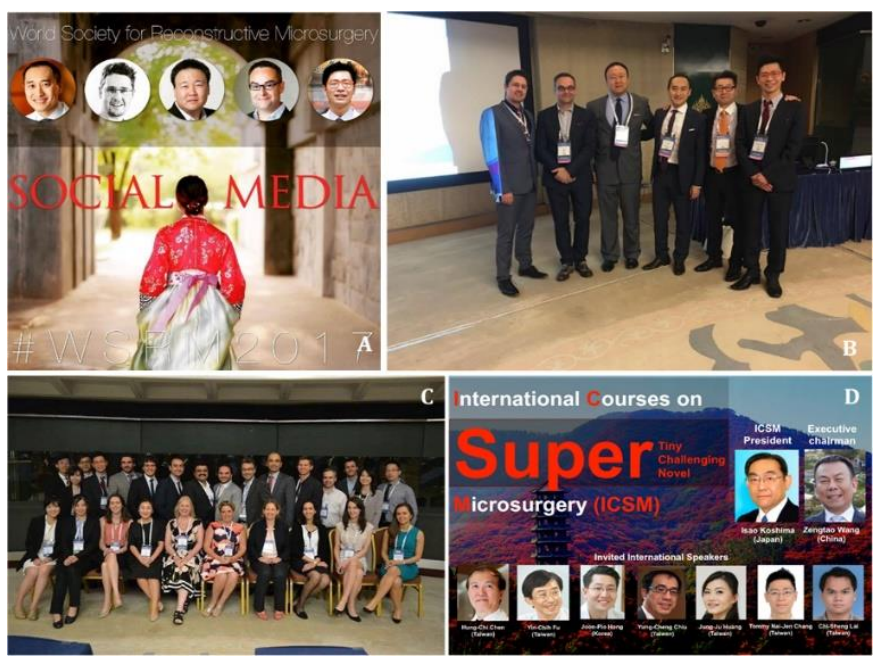

Figure 8. Social Media section has become increasingly popular in the International Microsurgery meeting. (A) World Society Reconstructive Microsurgery (WSRM) meeting in Seoul, Korea, Jun 2017. (B and C) International Microsurgical Simulation Society (IMSS) meeting in Seoul, Korea, June 2017. (D) International Courses on Super Microsurgery (ICSM) meeting in Jinan, China, October 2017.

Received: Aug. 25, 2017; Accepted: Oct. 16, 2017; Published: Dec. 7, 2017

DOI: $10.24983 /$ scitemed.imj.2017.00045

Copyright $\odot 2017$ The Author (s). This is an open-access article distributed under the terms of the Creative Commons Attribution 4.0 International License (CC-BY).

Funding: This research did not receive any specific grant from funding agencies in the public, commercial, or not-for-profit sectors.

Conflict of Interest: The authors declare no financial and personal relationships with other people or organizations with any interest financial or otherwise in the subject matter discussed in this manuscript.

\section{Keywords}

Social media; International Microsurgery Club; medical education. 


\section{LETTER}

\section{References}

1. Sutherland S, Jalali A. Social media as an open-learning resource in medical education: current perspectives. Advances in Medical Education and Practice 2017;8:369-375.

2. Cheston CC, Flickinger TE, Chisolm MS. Social media use in medical education. Academic Medicine 2013;88:893-901.
3. Long X, Qi L, Ou Z, et al. Evolving use of social media among Chinese urologists: opportunity or challenge? PLOS One 2017;12:1-11.

4. International Microsurgery Club. Available at: https://www.facebook.com/groups/1702063276733451/

5. Chang TN-J. Knowledge Revolution through Online Interactive Platform: International Microsurgery Club. International Microsurgery Journa/2017:1:2-3. 